PoS $\quad \begin{aligned} & \text { PROCEEDINGS } \\ & \text { OF SCIENCE }\end{aligned}$

\title{
Probing the Quark Sea and Gluons: the Electron-Ion Collider Projects
}

Rolf Ent*Jefferson Lab, Newport News

E-mail: entejlab.org 


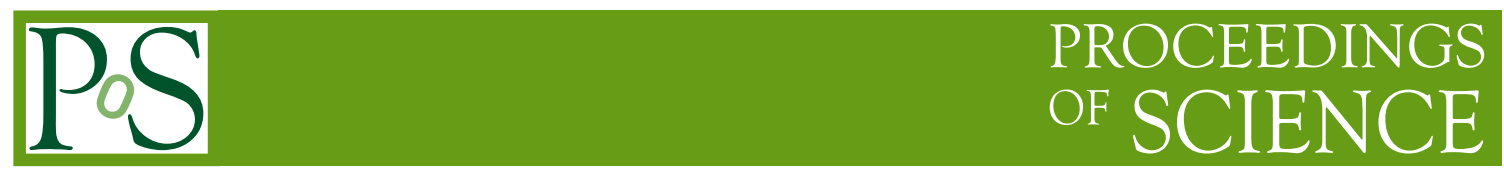

EIC is the generic name for the nuclear science-driven Electron-Ion Collider presently considered in the US. Such an EIC would be the world's first polarized electron-proton collider, and the world's first $e-A$ collider. Very little remains known about the dynamical basis of the structure of hadrons and nuclei in terms of the fundamental quarks and gluons of Quantum Chromodynamics (QCD). A large community effort to sharpen a compelling nuclear science case for an EIC occurred during a ten-week program taking place at the Institute for Nuclear Theory (INT) in Seattle from September 13 to November 19, 2010. The EIC science case and the initial detector design ideas are well documented in a report on this joint BNL/INT/JLab program [1].

The critical capabilities of a stage-I EIC are a range in center-of-mass energies from 20 to 70 $\mathrm{GeV}$ and variable, full polarization of electrons and light ions (the latter both longitudinal and transverse), ion species up to $A=200$ or so, multiple interaction regions, and a high luminosity of about $10^{34}$ electron-nucleons per $\mathrm{cm}^{2}$ and per second. The physics program of such a stage-I EIC [2] encompass inclusive measurements $\left(e p / A \rightarrow e^{\prime}+X\right)$, which require detection of the scattered lepon and/or the full scattered hadronic debris with high precision, semi-inclusive processes $\left(e p / A \rightarrow e^{\prime}+h+X\right)$, which require detection in coincidence with the scattered lepton of at least one (current or target region) hadron; and exclusive processes (ep $/ A \rightarrow e^{\prime}+N^{\prime} / A^{\prime}+\gamma / m$ ), which require detection of all particles in the reaction.

The main science themes of an EIC are to i) map the spin and spatial structure of quarks and gluons in nucleons, ii) discover the collective effects of gluons in atomic nuclei, and (iii) understand the emergence of hadronic matter from color charge. In addition, there are opportunities at an EIC for fundamental symmetry and nucleon structure measurements using the electroweak probe. To truly make headway to image the sea quarks and gluons in nucleons and nuclei, the EIC needs high luminosity over a range of energies as more exclusive scattering probabilities are small, and any integrated detector/interaction region design needs to provide uniform coverage to detect spectator and diffractive products. This is because $e-p$ and even more $e-A$ colliders have a large fraction of their science related to what happens to the nucleon or ion beams.

As a result, the philosophy of integration of complex detectors into an extended interaction region faces challenging constraints. Designs feature crossing angles between the protons or heavy ions during collisions with electrons, to remove potential problems for the detector induced by synchrotron radiation. Designs allocate quite some detector space before the final-focus ion quads, at the cost of luminosity, given that uniform detection coverage is a must for deep exclusive and diffractive processes. The integrated EIC detector/interaction region design at JLab focused on establishing full acceptance for such processes over a wide range of proton energies (20-100 GeV) with well achievable interaction region magnets. The detector design at BNL uses the higher ion beam energies to achieve good detection efficiency for instance for protons following a DVCS reaction, for proton beam energies starting from $100 \mathrm{GeV}$. Following a recommendation of the 2007 US Nuclear Science Long-Range Planning effort, the DOE Office of Nuclear Physics (DOE/NP) has allocated accelerator R\&D funds to lay the foundation for a polarized EIC. BNL, in association with JLab and DOE/NP, has also established a generic detector R\&D program to address the scientific requirements for measurements at a future EIC.

Sixth International Conference on Quarks and Nuclear Physics

April 16-20, 2012

Ecole Polytechnique, Palaiseau, Paris

\footnotetext{
*Speaker.
} 


\section{Introduction}

Understanding the fundamental structure of matter in the visible universe, and its evolution from its Big Bang origin to its current state, are amongst the most central goals in the pursuit of human scientific knowledge. This evolution is intimately tied to the properties of the particles that existed in each of its phases, ultimately leading to the structures and interactions of the visible matter around us, responsible for people and stars. The structure of visible matter appears simple, the mass of an atom is completely dominated by the mass of the nucleus, and in turn by the mass of the nucleons (neutrons and protons) within it. However, it is the internal structure of these nucleons and nuclei on the basis of the fundamental theory of strong interactions, Quantum ChromoDynamics (QCD), that is the key to understanding the dynamical origin of mass in the visible universe and the behavior of matter at astrophysical temperatures and densities.

Even if there remain large unknowns in understanding their properties and behavior, that socalled valence quarks are relevant to nucleon structure is well known. Valence quarks are key ingredients of the nucleon, and responsible for their charge, baryon number and other macroscopic quantum numbers. Over the previous two decades, it was found however that both sea quarks and gluons also play an unexpected prominent role in nucleon structure, with $99 \%$ of the proton's mass due to the self-generating gluon field and the proton sea having a non-trivial structure. Experiments at FNAL uncovered that the light quark sea of up and down quarks is an integral part of nucleon structure. Experiments at DESY found that gluons largely dominate the nucleon's internal momentum distribution for momenta smaller than $10 \%$ of the full nucleon momentum. The nucleon is now well known to be far more than its simple three-quark valence structure, yet the exact roles of the quark sea and gluons to nucleon (and nuclear) structure remain unknown.

The ultimate tool to discover the impact of the quark sea and the abundant gluons on nucleon and nuclear structure is well recognized to be an Electron-Ion Collider, with polarized electron and nucleon beams in combination to acces to nuclear beams up to $A=200$ or so. A collider environment provides, beyond its energy reach, tremendous advantages to make dramatic progress in terms of for instance polarization flexibility and purity, and access to spectator quarks, as compared to fixed-target facilities. The Electron-Ion Collider would be the world's first polarized electronproton collider and the world's first $e-A$ collider, and shed light on the dynamical basis of the structure of hadrons and nuclei in terms of the fundamental quarks and gluons of QCD. In particular, the main science themes [2] of an Electron-Ion Collider are to i) map the multi-dimensional spin and spatial structure of quarks and gluons in nucleons; ii) discover the collective effects of gluons in atomic nuclei; and iii) understand the emergence of hadronic matter from color charge. In addition, fundamental symmetry measurements are foreseen using the electroweak probe.

Worldwide, there are four electron-ion collider projects under study:

- The Large Hadron-electron Collider (LHeC) at CERN. An extensive report on the physics and design concepts for machine and detector by the LHeC Study Group just appeared [3]. The $\mathrm{LHeC}$ would add a newly built electron beam of $60 \mathrm{GeV}$, to possibly $140 \mathrm{GeV}$, to collide with the intense highly-energetic hadron beams of the LHC. Compared to the first $e-p$ collider, HERA at DESY, the kinematic range is extended by a factor of twenty, while the integrated luminosity is projected to be trumped by two orders of magnitude. The LHeC thus continues the path of deep inelastic scattering into unknown areas of physics and kinematics, 
and has a kinematic reach unprecedented to investigate a variety of fundamental questions in strong and electroweak interactions. The $\mathrm{LHeC}$ would already with $e-p$ beams have tremendous reach to study the initial conditions of Quark-Gluon Plasma formation.

- The Electron-Nucleus Collider (ENC) at GSI/FAIR. The ENC project aims to realize an electron-nucleus collider at the upcoming facility for Antiproton and Ion Research FAIR at GSI Darmstadt [4]. It foresees the addition of a $3.3 \mathrm{GeV}$ storage ring for polarized electrons to the planned $15 \mathrm{GeV}$ proton beam, or $7.5 \mathrm{GeV}$ deuteron beams. The reach in center-ofmass energy is modest, up to about $14 \mathrm{GeV}$, with the main (nuclear) science goal for the ENC that of multi-dimensional parton imaging in the sea and valence quark region.

- The US-based Electron-Ion Collider (EIC), with designs ongoing at both BNL to add an electron beam to the existing RHIC tunnels (eRHIC) and at JLab to augment the $12-\mathrm{GeV}$ Upgraded CEBAF with a hadron beam facility (MEIC). Extensive design reports for both eRHIC and MEIC exist, and are available from the various web pages [5]. The EIC foresees both highly polarized light-ion (longitudinally and transversely polarized) and unpolarized heavy-ion beams up to $\mathrm{A}=200$ or so from the start. The main science themes of the USbased EIC are closely related to the topics of Quarks in Nuclear Physics and will be expanded upon in more detail below (see also [1, 2, 6]).

\section{The Role of the Quark Sea and Gluons in Nucleon and Nuclear Structure}

Today, we stand at the frontier of understanding precisely how the quarks and gluons are assembled to form hadrons and nuclear matter in the framework of QCD. This is one of the central problems of modern nuclear physics, and the key to understanding the dynamical origin of mass in the visible universe and the behavior of matter at astrophysical temperatures and densities. It is an essential element in describing nuclear structure and reactions from first principles, a project with numerous potential applications to science and technology. Theoretical methods to apply QCD to hadronic and nuclear systems have made dramatic advances in the last two decades but rely crucially on new experimental information for further progress.

Electron scattering has been well established as a powerful tool for exploring the structure of matter at the sub-femtometer level $\left(<1 \mathrm{fm}=10^{-15} \mathrm{~m}\right)$. Historically, such experiments provided the first proof of the extended nature of the proton and revealed the presence of pointlike constituents, or quarks, at smaller scales, revolutionizing our understanding of strong interactions. Subsequent experiments established the validity of QCD and the presence of gluonic degrees of freedom at short distances and measured the basic number densities of quarks and gluons in the nucleon (proton, neutron). Surprisingly, high-energy experiments also measured an asymmetry in the light quark sea of up and down quarks, establishing the relevance of the quark sea to nucleon structure. While much progress has been made, several key questions remain unanswered [2]:

1. What role do sea quarks and gluons play in nucleon structure? What are their spatial distributions, and to what extent do they differ? How do they respond to polarization? What is their orbital motion, and how does it contribute to the nucleon spin? The answers to these questions will provide essential information on the effective degrees of freedom emerging from QCD at distances of the order of the hadronic size $(\sim 1 \mathrm{fm})$. 
2. What are the properties of the fundamental QCD color fields in nuclei with nucleon number A $>1$ ? What are the nuclear gluon and sea quark densities that are to date unknown? To what extent are they modified by nuclear binding, quantum-mechanical interference, and other collective effects? These questions are the key to understanding the QCD origins of the nucleon-nucleon interaction, the role of non-nucleonic degrees of freedom, and the approach to a new regime of high gluon densities and saturation at high energies.

3. How do colorless hadrons emerge from the colored quarks and gluons of QCD? What dynamics governs color neutralization and hadron formation? By what mechanisms does the color charge of QCD interact with nuclear matter? Even though Einstein's famous law of $M=E / c^{2}$ is at the root of modern physics, we are still far from understanding the fundamental processes by which hadronic matter is created from high-energy radiation.

To illustrate the scientific potential of the EIC to give concrete answers to these key questions, some examples of measurements are now given, which by no means represent the complete rich science program foreseen. First of all, the polarized EIC could give decisive measurements on how much the intrinsic spin of quarks and gluons contribute to the proton spin [7]. Present uncertainties on quark spin contributions to the proton spin would be reduced by a factor of two, uncertainties on gluon spin contributions by about a factor of ten. Measurements of various exclusive processes, in which all collision products are detected, possible at an EIC due to its unprecedented luminosity and detector coverage, would allow for transverse images of the proton in terms of the distributions of quarks (including various quark flavors) and gluons [8]. Recent work has shown that EIC measurements would allow for these distributions all the way from the center of the proton to a transverse distance as far as $1.5 \mathrm{fm}$ [9]. At such large distance scales the QCD interactions are strong, and aspects of proton structure intimately connected with the dynamics of color confinement would be revealed. Alternately, the EIC with its collider energies and polarized beams will be able to selectively probe the correlation between the spin of a fast moving proton and the confined transverse motion of quarks and gluons inside the proton. This will allow one to extract analogous images of the (quarks and gluons inside the) proton in momentum space [10]. These measurements together will revolutionize our understanding of the proton internal wave function, and transcend from the present limited one-dimensional views of sea quarks and gluons inside the proton to a three-dimensional view (as function of Bjorken $x$ ), sensitive to orbital motion.

Secondly, understanding on the structure and dynamics of the QCD color fields in nuclei, and how these fields differ from the sum of the color fields of the individual nucleons, can be obtained by studying the scattering of small-size probes - e.g., a virtual photon with $Q^{2} \gg 1 \mathrm{GeV}^{2}$ - from nuclei over a range of incident energies. The lifetime of the probe in the target rest frame is defined by the coherence length. If the coherence length is much smaller than the nuclear radius, the scattering involves only a single nucleon in the nucleus. In this regime the results can be interpreted in terms of a modification of single-nucleon structure through nuclear binding and reveal the QCD origins of the nucleon-nucleon interaction. If the coherence length becomes comparable to or larger than the nuclear radius, the color field seen by the probe is the quantum-mechanical superposition of the fields of the individual nucleons, resulting in a rich spectrum of coherence effects such as shadowing, diffraction, and eventually the approach to the unitarity limit (saturation) at high energies [2]. Nuclear deep-inelastic scattering with an EIC would also for the first time allow one 
to determine the gluon and sea quark densities in a range of nuclei, penetrating thanks to the wide kinematic coverage deep into the shadowing region - a step that might prove as revolutionary for our understanding of nuclei as the discovery of the quark EMC effect 30 years ago.

Lastly, the emergence of colorless hadrons from the elementary color charge produced by short-distance probes - the so-called hadronization process - is a principal aspect of QCD which still lacks a quantitative understanding from first principles. Knowledge of the underlying dynamics remains sketchy and model-dependent. By measuring the energy loss and transverse momentum broadening of leading hadrons induced by the nuclear environment one can discriminate the different dynamical processes and infer the space-time evolution of hadronization. The combination of high energy and luminosity offered by the EIC promises a truly qualitative advance in this field, where the collider environment would allow simultaneous study of the evolution of the system from which a color charge has been removed by the hard process. In this way one could also follow the materialization of the color hole in the nucleon created by the hard process, and measure particle correlations between the struck quark and color hole.

\section{The Electron-Ion Collider: Integrated Detector and Interaction Region Designs}

The envisioned EIC will serve well-established scientific user demand by colliding electrons (and possibly positrons) with polarized protons and with light to heavy nuclei at high energies, offering unprecedented opportunities for in-depth investigations of QCD. Physics studies have shown that with a luminosity of close to $10^{34} / \mathrm{cm}^{2} / \mathrm{s}$, and varying electron and ion beam energy, polarization and ion species configurations, this EIC will support a wealth of groundbreaking experiments. Multi-dimensional study of nuclear processes will require about a factor of 1,000 more integrated luminosity than recorded over a decade at the only previous electron-proton collider, HERA at DESY in Hamburg, Germany, at least to recognize the more diverse beam conditions required.

The most important design requirements of the envisioned EIC are:

- Highly polarized ( $>70 \%)$ electron and nucleon beams, with both electron and nucleon beams longitudinally polarized and with nucleon beams transversely polarized as well.

- Ion species from deuterium to $\mathrm{A}=200$ or so.

- Center of mass energies from $\sqrt{s} \sim 20$ to $70 \mathrm{GeV}$ and variable, with electron and proton energy adjustable to optimize particle identification and reach in Rosenbluth separations.

- Upgradeable to center of mass energy of $\sqrt{s} \sim 150 \mathrm{GeV}$.

- Multiple interaction regions.

- High luminosity: $\approx 10^{34}$ e-nucleons $/ \mathrm{cm}^{2} / \mathrm{s}$.

- Integrated detector/interaction regions to maximize detector acceptance and resolution.

To meet these requirements, two collider design efforts are underway in the U.S., one based on an existing accelerator at Brookhaven National Laboratory and one based on the $12 \mathrm{GeV}$ upgrade of the Continuous Electron Beam Accelerator Facility (CEBAF) now in progress at Thomas Jefferson 
National Accelerator Facility. At BNL, the eRHIC design calls for beams from a new linear electron accelerator to collide with the polarized proton and ion beams of the existing Relativistic Heavy Ion Collider (RHIC). At JLab, the MEIC (Medium energy Electron Ion Collider) design calls for new electron and ion storage rings to operate with $12 \mathrm{GeV}$ CEBAF. In both cases, $\mathrm{R} \& \mathrm{D}$ will be required, notably for the hadron-beam cooling necessary to reach the needed luminosities. Roughly, eRHIC uses the existing $250 \mathrm{GeV}$ proton beams to collide with new 5-10 GeV electron beams, whereas MEIC uses the $12 \mathrm{GeV}$ upgraded electron beam to collide with new 20-100 GeV proton beams, to establish a similar reach in center of mass energy $\sqrt{s}$.

The interaction region, acting as an interface between the beam acceleration/transport system and the detector, is one of the most challenging parts of the EIC designs, and it largely determines the science performance of the collider. Due to the large asymmetry in the electron (5-10 GeV) and ion beam energies, roughly a decade higher, the layout of the interaction region design is asymmetric. Much of the science of an electron-ion collider is associated with the detection of spectator quarks and nuclear fragments moving along the ion beam direction in coincidence with hadrons originating from the struck partons. Thus, special emphasis is placed on the ion beamline elements downstream of the interaction point in an effort to measure all the particles in the event with good resolution, including the high-momentum ones emerging at small angles. To meet the nuclear physics requirements, the integrated detector and (far extended) interaction region needs to be able to "peel" these fragments away from the ongoing beam, without causing gaps in detection.

The detector and extended interaction region needs to fulfil the following requirements:

1. Hermeticity To allow for detection of scattered electrons, mesons, and baryons - charged and neutral - without holes in the acceptance, even in forward and backward regions.

2. High-luminosity capability To allow operation in a high-luminosity environment with moderate event multiplicities.

3. Acceptable background conditions Placement of the detector and specific beam geometries should minimize synchrotron radiation, and residual gas detector backgrounds.

4. Beam transport and manipulation Care must be taken off all magnetic elements encompassing the detector, including additional magnetic elements in forward and backward region, which will often serve double duty as spectrometer magnet and beam optics magnet.

These requirements lead to layouts of the detector and extended interaction region that may seem counterintuitive. For instance, final focusing quads are positioned relatively far from the interaction point, about five to seven meters, at the cost of luminosity (focusing) but gaining resolution and minimizing holes in the acceptance. Designs feature crossing angles between the protons or heavy ions during collisions with electrons, with the electron beam traversing the central axis of the detector magnets without deviation to minimize synchrotron backgrounds. More up and downstream magnetic elements serve double duty, with gaps and spacings between magnetic elements suffcient to "squeeze" small-angle patricles through the magnet gaps and subsequently detect them. This without putting overly large demands of quadrupole gradients or peak fields, preferentially limited to $6 \mathrm{~T}$ or so. More considerations can be found in [11, 12, 1, 5]. The MEIC interaction region layout, for example, has been designed to fulfill these requirements and achieves an unprecedented hermetic detection with essentially no holes in acceptance. 


\section{Outlook}

The proposed US-based Electron-Ion Collider was designated in the 2007 Nuclear Physics Long Range Plan as "embodying the vision for reaching the next QCD frontier". Since that time, through a large world-wide community effort coupled with recent progress and excitement that we are on the verge of a dramatic improvement of understanding of the role of quarks and gluons in nucleon structure and nuclear physics, the science case of a future EIC has drastically grown.

In parallel, designs for such an EIC have matured at both BNL and JLab, with much attention paid to allow for (i) high luminosity to be able to detect so-called deep exclusive process that come with small scattering probabilities, as spearheaded at DESY and JLab, (ii) high proton beam polarization in both longitudinal and transverse direction following the unique successes at BNL, (iii) flexibility in energies and ion species up to heavy nuclei, and (iv) full integration of the detector and extended collider interaction region to establish complete detector acceptance. Research and design efforts for critical accelerator science requirements for a future EIC, and associated R\&D efforts for detector systems related to an EIC, have been established and are well on their way.

Significant theoretical and experimental progress has been made over the past decade to pave the road towards a true understanding of nucleons and nuclei - their structure and origin, in terms of the basic constituents, quarks and gluons. It has become more and more clear that an electronion collider is the experimental tool required to allows this breakthrough. At this stage, we know enough to define the capabilities of such an EIC, and these parameters are commonly endorsed by the wide nuclear physics community. We are eagerly awaiting full approval for realization of such an EIC after seeking recommendation at the next US Nuclear Physics Long Range Planning effort, anticipated around 2014.

\section{References}

[1] D. Boer, R. Milner, R. Venugopalan, W. Vogelsang et al., arXiv:1108.1713 [nucl-th] (2011).

[2] A. Accardi, V. Guzey, A. Prokudin, and C. Weiss, Eur. Phys. J. A 48 (2012) 92; arXiv:1110.1031 [hep-ph] (2011).

[3] J. L. Abelleira Fernandez et al. [LHeC Study Group Collaboration], arXiv:1206.2913 [physics.acc-ph] (2012).

[4] A. Lehrach et al., J. Phys. Conf. Ser. 295, 012156 (2011).

[5] For further information and updates on the EIC machine designs, see: https://eic.jlab.org/wiki/ (JLab) and https://wiki.bnl.gov/eic/ (BNL).

[6] A. Deshpande, R. Milner, R Venugopalan and W. Vogelsang, Ann. Rev. Nucl. Sci. 55, 165 (2005).

[7] E. C. Aschenauer, R. Sassot and M. Stratmann, arXiv:1206.6014 [hep-ph] (2012).

[8] T. Horn. P. Nadel-Turonski and C. Weiss, arXiv:0908.1999 [hep-ph] (2009).

[9] M. Diehl, arXiv:1206.0844 [hep-ph] (2012).

[10] M. Anselmino et al., Eur. Phys. J. A 47, 35 (2011); arXiv:1101.4199 [hep-ex] (2011).

[11] T. Horn, Proc. of 4th Workshop on Exclusive Reactions at High Momentum Transfer (ed. A. Radyushkin), http://www.worldscibooks.com/physics/7998.html (2011).

[12] C. Hyde, R. Ent, P. Nadel-Turonski and V. S. Morozov, Conf. Proc. C 1205201, 2011 (2012). 